\title{
Scepticism towards insecticide treated mosquito nets for malaria control in rural community in north-western Tanzania
}

\author{
SOORI E. NNKO, SUSAN R. WHYTE, WENZEL P. GEISSLER and JENS AAGAARD-HANSEN \\ ${ }^{1}$ National Institute for Medical Research, Mwanza Research Centre, P.O. Box 1462, Mwanza, Tanzania \\ ${ }^{2}$ Department of Anthropology, Faculty of Social Sciences, University of Copenhagen, Denmark \\ ${ }^{3}$ London School of Hygiene and Tropical Medicine, Keppel Street, London, UK \\ ${ }^{4}$ Steno Diabetes Center A/S, Niels Steensens Vej 8, DK-2820 Gentofte, Denmark
}

\begin{abstract}
Despite existence of effective tools for malaria control, malaria continues to be one of the leading killer diseases especially among under-five year children and pregnant women in poor rural populations of Sub Saharan Africa. In Tanzania Mainland the disease contributes to $39.4 \%$ of the total OPD attendances. In terms of mortality, malaria is known to be responsible for more than one third of deaths among children of age below 5 years and also contributes for up to one fifth of deaths among pregnant women. This paper is based on a study conducted in a rural community along the shores of Lake Victoria in Mwanza region, North-Western Tanzania. The study explores reasons for scepticism and low uptake of insecticide treated mosquito nets (ITNs) that were promoted through social marketing strategy for malaria control prior to the introduction of long lasting nets (LLN). The paper breaks from traditional approach that tend to study low uptake of health interventions in terms of structural practical constraints - cost, accessibility, everyday priorities - or in terms of cognition insufficient knowledge of benefits e.g. ignorance of public health messages. This paper has shown that, the majority of people who could afford the prices of ITNs and who knew where to obtain the insecticides did not necessarily buy them. This suggests that, although people tend to report costrelated factors as a barrier against the use of ITNs, there are other critical concerns at work. Without underestimating the practical factors, our study have recommended to consider critical examinations of those other concerns that hinder optimal utilization of ITN for malaria control, and the basis for those concerns.
\end{abstract}

Key words: scepticism, low uptake, mosquito nets, malaria, social marketing, Tanzania

\section{Introduction}

Since mid 1990s, Tanzania government through public private partnerships approach has invested substantial resources to promote insecticide treated mosquito nets (ITNs) as an integral strategy for control of malaria vector in the country. As a strategy to scale up the use of ITN, malaria control programme has actively employed social marketing approach, such as subsidized voucher scheme for mosquito nets distribution and re treatment among the most vulnerable groups for malaria such as pregnant women and under-five years' old children. Several studies conducted in the country have shown that, despite aggressive promotion of ITN, many household members did not own ITN, and even those who owned it did not consistently sleeping under ITNs. For example, some household members in Bagamoyo, Tanzania who were provided subsidised mosquito nets did not sleep under the nets (Winch et al.. 1994, 1997). Studies conducted elsewhere in East Africa have similarly shown that, even when the nets are provided for free, substantial percentages of people did not use them (Alaii et al., 2003).

Ambivalence towards public health intervention is not a new phenomenon. Historical records indicate that biomedical interventions during colonial period, such as small pox vaccination in Malawi (Vaughan, 1991) and treatment of sleeping sickness in Uganda (Hoppe, 1997) and Tanzania (Kjekshus, 1977) were also received with scepticism. 
More recently in Sub-Saharan Africa, there has been an upsurge of reports about suspicions towards modern family planning methods (Wolf, 1985), and sexual and reproductive health interventions such as promotion of male condoms for prevention against HIV infections (Bledson et al., 1998; Feldman-Savelsberg et al., 2000; Maharaj, 2001). Indeed vaccination programmes have been one of the most resisted biomedical interventions not only in African but also in East Asian countries (Nichter \& Nichter, 1996; Bierlich, 2000) and even in Europe (Leach \& Fairhead, 2007).

Scepticism towards interventions to improve health is manifested in different forms, such as expressing mistrust, foot-dragging, rumour or outright resistance to the intervention (Leach \& Fairhead, 2007). Whyte et al. (2002) have suggested that people's scepticism to medical interventions may be caused by their concerns about the toxicity of medical technology e.g. medicinal substances, while others 'may resist the aggressive and debilitating effects of medicine on the body's immunity'. A study conducted in Brazil found that people were ambivalent about pharmaceuticals, mainly because they associated pharmaceuticals with modernity, which has disappointed people in other areas of their life (Wayland, 2004). The view that scepticism towards pharmaceuticals can be understood as a critique of modernity is also shared by Whyte et al. (2002), who have argued that, 'sometimes objections to medicines are phrased in terms of what might be called cultural idioms, where biomedical drugs are compared unfavourably to natural medicines or indigenous medicines.'

Despite prevailing resistances to various health interventions, there is evidence that people are also attracted towards medical technologies (Birungi, 1998; Whyte et al., 2002). Some scholars have for instance shown that given the increased popularity of pharmaceuticals in non-western societies, biomedicines have transcended professional health sector's boundaries, thus allowing lay people to handle and exchange prescription medicines - on a commercial basis or as a token (Birungi, 1998).

There are genuine concerns however that most debates about and explanations for people's responses to public health interventions and particularly medical technologies are framed in terms of public misunderstanding or lack of understanding of science, technology or its risks. Review by McCombie (1996) of various social science researches conducted in Africa to understand why local people did not adhere to recommended malaria control measures or biomedical treatment unanimously suggested that people had inadequate knowledge on the interventions which led them to act contrary to what was advocated by the biomedical practitioners. Another systematic review of qualitative studies conducted more recently, have similarly linked low uptake of malaria interventions during pregnancy with inadequate knowledge of symptoms and aetiologies of malaria, and also inadequate awareness about ante-natal care services and negative attitudes towards health workers (Pell et al., 2011).

The 'deficit paradigm' tends to suggest that the 'lack' may be not just of knowledge, but also of trust - in both science itself and in its governance (Leach \& Fairhead, 2007). Leach and Fairhead (2007) have underscored that the overemphasis on what people do not understand, misses the opportunity to identify the 'framing' - forms of knowledge, value and social commitment - that people bring to an issue, and which shape their anxieties about it, whether positive or negative. And it misses opportunities to identify mismatches between people's framings, and those of the institutions involved with science or governance. Accordingly, they recommend a 'positive perspective' that focuses not only on 
how the public frames issues involving the proposed medical technologies but also on how the institutions or authorities promoting the technology frame it.

This paper breaks from the traditional approach that tend to regard structural constraints as the major determinant impediment for the uptake of health intervention e.g. poor income, ignorance of public health messages, and unavailability and accessibility to the intervention. The paper provides alternative approach in understanding the low/ or delayed uptake of effective interventions for malaria control by interrogating the relations through which the ITNs come into being and through which the products were distributed - notably the realms of government and the market - and it situates people's experience of illness and health intervention within their wider engagement with the political economy. Without underestimating the practical factors, this paper aims to situate scepticism which negatively affects the uptake of ITN into wider social-cultural and political contexts in which ITN interventions were introduced.

\section{Material and Methods}

\section{Study area and population}

This paper is based on cumulative experience from ethnographic fieldwork coupled with household survey which took place from 2003-2008 in five villages of Busisi ward in Sengerema District of north-western Tanzania. Two of the 5 villages (Kahumlo and Lubanda) are typically land locked rural settlements, whereas the rest villages (Nyamasale, Nyitundu and Mkomba) are located along the Mwanza to Geita roadside bordering the shores of Lake Victoria.

\section{Data collection tools}

To collect qualitative data, three main techniques were used, namely: In-Depth Interviews (IDI), Focus Group Discussions (FGD), and Participant Observation (PO). In total we conducted 13 FGDs with study participants who were drawn from different social categories, including ward' extension workers from various departments (i.e. Health, Education, Livestock Development, Welfare Development, Fisheries and Forestry). We similarly held FGDs with influential village leaders, opinion leaders and purposively selected heads of households.

We also conducted fifty two In-Depth Interviews (IDIs) with influential village elders or opinion leaders, health workers, heads of households and village leaders. During the entire period of the fieldwork we also conducted Participant Observations in formally organised social gathering as well as in the informal social gathering. The sites visited for POs included school compounds, health facility settings and in drug shops, weekly mobile markets (gulio) the community. Since the fieldwork involved prolonged stay, we were integrated into community and therefore had to equally play social roles including visiting families with malaria patients. During our visits we managed to observe aspects relevant to malaria control, including bedding facilities.

Quantitative data were collected through close ended questionnaire that were administered among 446 heads of households. Respondents for the questionnaire survey were randomly selected from a list of all heads of households that was provided to us by the local government authority. The heads of households were asked information about their knowledge and awareness on ITN and also about the actions or preventive measures that they themselves and other members of household took to prevent against malaria. Further 
detail regarding amount of people participated in IDI and Questionnaire survey, and number of sessions held for FGDs is presented in Table 1.

Table 1: Breakdown of number of interviews conducted

\begin{tabular}{lll}
\hline Data collection techniques & Category of participants & Number \\
\hline IDIs & Heads of households with treated bed nets & 15 \\
& Heads of households with untreated bed & 12 \\
& nets & \\
& Village leaders & 10 \\
& Opinion leaders & 9 \\
& Health staff workers & 6 \\
& Sub-total & \\
& Technical personnel from the study area & 2 \\
FGD & Opinion leaders & 3 \\
& Village leaders & 2 \\
& Health staff workers & 2 \\
& Heads of households with ITNs & 2 \\
& Heads of households without ITNs & 2 \\
& Sub-total & \\
Questionnaires interviews & Randomly selected heads of households & 446 \\
\hline
\end{tabular}

\section{Data processing and analysis}

Audio files from the IDIs and FGDs were verbatim transcribed, translated from Kiswahili to English and later thematically coded for interpretations. Quantitative data from questionnaire survey were double entered into computer using STATA software and followed by simple descriptive tabulations /analysis. Audio files from the Interviews and discussions were verbatim transcribed, translated from Kiswahili to English and later thematically coded for interpretations.

\section{Ethical Consideration}

Ethical approval was obtained from Medical Research Coordination Committee of the National Institute for Medical Research. Information on the study protocol was adequately shared with all study participants before their enrolment to the study. Interviews with participants were only conducted after receiving verbal informed consent from each participant.

\section{Results}

To get an estimate of proportion of people sleeping under ITNs, we administered questionnaire to 446 heads of household, which explored measures that each of the household members used to protect themselves against mosquitoes a night before the interview. The 446 head of households provided information about preventive measured used by a total of 2219 people who slept in their houses a day before the interviews. From the survey it was found that, the majority (52\%) of household members slept under untreated mosquito nets, followed by $39.2 \%$ who did not use any measures. Only 2.7 of respondents slept under treated bed nets (Table 2). 
Table 2: Preventive measures taken against mosquito bites $(\mathrm{n}=\mathbf{2 2 1 9})$

\begin{tabular}{lcc}
\hline Methods used to protect against mosquitoes last night? \\
\hline Attributes & Frequency & Percentages (\%) \\
Untreated bed nets & 1166 & 52.5 \\
Treated bed nets & 59 & 2.7 \\
Mosquito coils & 17 & 0.8 \\
Burn herbs & 6 & 0.3 \\
Keep windows \& doors closed & 100 & 4.5 \\
None & 871 & 39.2 \\
Total & 2219 & 100 \\
\hline
\end{tabular}

The questionnaire asked reasons for not sleeping under treated mosquito net $(n=188)$ and slightly less than half of the respondents (93/188) (49.5\%) claimed that treated mosquito nets were too expensive. Ten respondents (5.3\%) claimed that, although they were able to afford the price, they did not know where to buy mosquito insecticide in their village (Table 3 ).

Table 3: Reasons for not using treated mosquito nets $(n=188)$

\begin{tabular}{lll}
\hline Attributes & Frequencies & Percentages (\%) \\
\hline $\begin{array}{l}\text { Insecticide too costly } \\
\begin{array}{l}\text { Able to buy but lack supply of } \\
\text { insecticide }\end{array}\end{array}$ & 93 & 49.5 \\
Fear of harmful effects of insecticide & 3 & 5.3 \\
Doubted the efficacy of insecticides & 40 & 1.6 \\
Neglect & 35 & 21.3 \\
Other reasons & 7 & 18.6 \\
Total & $\mathbf{1 8 8}$ & 3.7 \\
\hline
\end{tabular}

According to the interview conducted with one district health official, the price of insecticides treated medium-size mosquito net $(4 \times 6$ feet) ranged from Tanzania Shillings (TShs) 3500 to 5000. She however indicated that, "[since] the government has allowed mobile vendors (wamachinga) to sell the nets, they can ask for higher prices than the officially approved ones" (Interview with female, nurse, from Sengerema District Hospital). Interview with mobile vendors who sold mosquito nets in the study area, confirmed that the prices of bed nets varied depending on sizes and shapes of the net, where the retail price for a rectangular medium-size mosquito net ranged between TShs 4000 to 6000 (US\$1=TShs. 1200).

\section{Perceptions towards toxicity of insecticides}

Most people reported fear that insecticide could cause health problems, including "chronic diseases". One respondent who had himself participated in social marketing seminar indicated that many people were reluctant to treat or re-treat mosquito nets because they feared that NGAO (commercial trade mark for the insecticide used to treat mosquito nets) could cause tuberculosis (TB). Other respondents claimed that when someone is covered with treated mosquito nets, "all the insecticide passes through the nose and causes lung diseases. [...] If the bedbugs die when they touch the treated mosquito net, it could also 
harm our children if they touch it" [Interview with primary school teacher]. The fear that ITNs were harmful is further exemplified by conversation between a shop keeper and a client that we observed during the fieldwork, which started after a one female shop client, had asked a shopkeeper to explain to her how to treat bed net with NGAO (insecticide). The following were responses from the shopkeeper: "Why are you asking [for clarification], [...], don't you listen to the radio [announcements]? It's safe and easy to use, [...], you have to soak it for few minutes, dry it and use it. But remember to wear the gloves. [...] There is also a piece of paper inside the sachet; [...] you can read it! [...] Maybe if you are asthmatic, it may cause you some problems. [...] I have never heard anyone complaining about it".

\section{Scarring public health campaigns}

Some respondents confessed that they did not treat mosquito nets with NGAO because of scaring messages from public health campaigns about insecticides. One opinion leader said that, the leaflets that promoted insecticides were written "sumu" (Kiswahili word, for poison). This caption scared people, because poison substances are dangerous. This is illustrated from the extract taken from an interview with opinion leaders: "[...] Those leaflets are marked with other scarring instructions such as: 'Keep away from children,' 'Do not swallow', 'If it accidentally comes into contact with your skin, wash it thoroughly.' Moreover, inside the packet, there are also pair of gloves that have to be worn to protect skin against contact with insecticides during re-treatment of the nets. According to the leaflets, after the re-treatment of mosquito nets, the gloves had to be discarded in a pit or burnt". [Female respondent, opinion leader, Mkomba village]

People believed that a person sleeping under treated mosquito net could inhale the poison. The extract below further illustrates the fear that insecticides may be dangerous to the health of people who use it:

Respondent: They gave me insecticide (dawa ya mbu) but I just put it inside the box. They say [that] if you apply it to a bed net, mosquitoes can't touch it, but I don't know if it's true. I have never tried it. They say insecticide is poisonous. So I fear it.

Interviewer: Who said it is poisonous? Where did you hear that?

Respondent: Just the people. I just heard it from other people. We are even instructed to cover our hands with plastic if we want to wash our nets with NGAO. [...] Mm, now if you are asked to cover the hands, what about yourself while inside the bed net? Mm! Myself I fear it. That is why I refused to treat my nets, [...] there is no other reason (A conversation between the researcher (Interviewer) and Respondent (Female respondent; influential person, Mkomba village).

Young children were perceived to be most susceptible to the 'insecticide poison'. As one of the villagers curiously asked our field assistant: '[W]e are told treated nets are able to kill mosquitoes for six months ... even ticks cannot survive if they land on it. [I]t must be so powerful, [...] what if our young children touch it?' Some though the insecticide was too poisonous to the extent that it could even harm the unborn baby if a pregnant woman inhales it. 
In contrast to respondents who believed insecticides were 'too powerful and harmful', a section of people who participate in the interviews perceived the ITN technology as a hoax. The sceptical respondent claimed that social marketing campaigns that promoted ITN were purely motivated by profit gains. According to them NGAO had no intrinsic powers to kill mosquitoes. An interview with a villager, who worked as village health worker (VHW) and who had been trained to promote and distribute ITNs in their community revealed that, some people questioned how a tiny tablet [referring to insecticide] could be able to protect people against mosquitoes bites for six months (ka-kidonge ka-dogo hivi kanawezaje kuzuia mbu kwa kipindi chote cha miezi sita?). One responded claimed that "mosquitoes have become familiar to the insecticide hence the NGAO doesn't kill them anymore".

\section{Discussion}

Using mixed data collection methods, this study has explores reasons for low uptake of insecticides mosquito net interventions that were promoted by the public and private sectors for malaria control in Tanzania. This study was conducted before the national up scaling of Long Lasting Insecticides Nets (LLINs). At the beginning of this study (in 2002), 70\% of the bed net' market in Tanzania that were sold through commercial channels was dominated by untreated nets, and the remaining 30\% by treated bed nets (Magesa et al., 2005). Since then, Ministry of Health together with other partners started aggressive campaigns to promote production and use of treated mosquito, including scaling up LLIN. The implications of interpretation of data from this study goes beyond ITN intervention, since it addresses chemical product whose utility and application (from ordinary man's point of view) is akin to indoor residual spraying (IRS) - intervention currently been promoted for malaria control in the Lake zone regions.

Inadequate income was mentioned as one of structural barrier to retreatment of mosquito nets. Several other studies conducted in the country prior to our study had similarly shown that cost related barriers are the leading barrier for uptake of health interventions. Some studies have even shown that in trial settings where the use of ITNs was high, the retreatment rate declined immediately after completion of the trials (Winch et al., 1994, 1997). A study conducted in Morogoro region in Tanzania has also shown that, despite considerable subsidies on insecticides, re-treatment rates remained low (ArmstrongSchellenberg et al., 2002). Those studies conducted prior to our study attributed low use of ITN with economic status (poor or lack of income), where the poorer were most likely to miss the ITN (Hanson et al., 2003). Contrary to the view that low income was key determinant of low uptake of treated mosquito nets, our study has shown that during the cotton harvesting seasons some households had enough money to buy and use ITN but they did not do so (data not shown). Likewise, survey with heads of households indicated that some household members who could afford the price, and who knew where to obtain insecticides did not use ITNs. These observations suggest that, apart from socio-economic factors, there were other pertinent reasons for not using ITNs.

Several studies have linked the low uptake of biomedical interventions with inadequate knowledge that is normally characterised by misconceptions towards proposed intervention among the targeted population. Lay perception about chemicals and toxicity were the key themes that constantly recurred in discussions about ITNs. Lay perceptions and discourse on insecticide provided important contexts for understanding responses towards ITN technology. From the point of view of local people, mosquito insecticides 
shared a number of characteristics with pharmaceuticals. Whereas in public health, dawa (medicines) are used to restore health, from the local people's point of view, it (dawa) can be used for both protection / restoration of health and destruction. Thus, the public health message on ITNs which says: 'ITNs can kill mosquitoes' fits well with local people's ideas about sumu (poison). This notion that ITNs kill, reinforced people's fear about the dangers of ITNs. Our study confirms findings from other studies from Tanzania which indicated that fear about toxicity of insecticides acted as key impediments for retreatment programme (Winch et al., 1997). Other workers in Kilombero, south-eastern Tanzania have similarly argued that people were scared by the word sumu (poison), which were printed on the cover of insecticide' sachet (Minja \& Obrist, 2005).

Although our study concur with other previous study that cognitive (inadequate knowledge, lay perception) and structural factors (low income) do negatively influence uptake of ITN for malaria control, this study has offered alternative paradigm by addressing the broader social cultural and political determinants that may equally shape people's uptake of new intervention. Our study proposes that much of what people understood about insecticides were shaped by their connections to the local as well as the national economical and political landscapes. People learn about insecticides and other malaria control interventions through social interactions with family members, friends and neighbours as well as shopkeepers and from local adverts. Our study has shown how transactions and the supply of mosquito nets in the study area involved myriads interactions with different actors at local and national levels: at local level they interacted with peddlers, and retail shop owners. This setup made the peddlers and local shop owners to act as brokers between local non-governmental organizations (NGOs), health sector and the state (the producers) on one hand and the local community on the other hand (the consumers). Accordingly, the local businessmen - peddlers and retail shop owners - were seen as representing the government and their collaborators (NGOs and private sector). Probably, because of the 'marriage' that existed between shopkeepers, peddlers on one hand and the government and private sector on the other any misdeed conducted by the traders (peddlers and shopkeepers) such as parachuting prices of commodities were perceived as to have been approved by the government.

Our study has shown that social marketing campaigns to promote increased uptake of ITNs was received with mixed feelings and reactions. Probably as some scholars have suggested, not all negative reactions to biomedical interventions should be associated with cultural factors. Studies that have over-privileged cultural factors have challenged for failing to pay enough attention to the political and economic determinants of compliance to biomedical interventions (Garro, 1986; Dein, 2007). According to Farmer (1999), although those studies may provide some useful information, they tend to shift the blame onto the people by exaggerating their agency. Farmer challenges researchers to acknowledge that adherence to biomedicine can be influenced by a complex array of factors, many of which are beyond the patient's control (Farmer, 1999).

Pels (1999) has offered suggestion on how to address complex factors which shape uptake of biomedical interventions. He argues, if we want to understand why people have suspicions about insecticides, their purpose, effect, origin and meanings, we need to situate them in the larger picture of people's experience of change, their memories, specifically of pharmaceutical technologies such as insecticides and medicines, but also of the history of government development policies and economic change. Our study has situated scepticism towards ITN to the changing Tanzanian political economy landscape. 
Using political economy approach, this paper has explored reasons and contexts for scepticism towards ITNs. The study suggests that cognitive factors such as misconceptions, have fuelled scepticism and subsequently affecting the retreatment of mosquito nets with insecticides and also acquisition of treated nets. It has also shown that income constraint was among the factor that was reported to hinder people from treating nets or purchasing treated nets. However, in contrast to what previous studies have claimed, our study has indicated that the cognitive and structural factors (income) only play superficial role. Instead, our study suggests that the scepticism towards ITNs and low uptake of treated nets was shaped by people's practical experiences and engagements into the changing political and economic landscapes of the country. Our paper interrogates the relations through which the insecticides and mosquito nets came into being and through which the products were distributed - notably the realms of government and the market - and it situates people's experience of health interventions within their wider engagement with the political economy. In the eyes of poor community members, the public-private 'marriage' adopted for delivery of health services (such as ITN) was seen as been purely motivated by profit motives, and therefore suspected.

\section{Acknowledgements}

We are very grateful to women and men from Busisi ward who accepted to participate in this study. We sincerely thank the Sengerema District Council and Busisi leaders for allowing this study to be conducted in their area. We enjoyed both administrative and technical support offered by the National Institute for medical research to researchers and team members of this study. This study was funded by Melinda and Bill Gates Foundation \& Danish Bilharziasis Laboratory, University of Copenhagen. Ethical clearance was provided by Medical Research Coordination Committee.

\section{References}

Alaii, J.A., Hawley, W.A., Kolczak, M.S., Kuile, F.O.T., Gimnig, J.E., Vulule, J.M., Odhacha, A., Oloo, A.J., Nahlen, B.L. \& Phillips-Howard, P.A. (2003) Factors affecting use of permethrin-treated bed nets during a randomized controlled trial in western Kenya. American Journal of Tropical Medicine and Hygiene 68, 137-141.

Bierlich, B. (2000) Injections and the fear of death: an essay on the limits of biomedicine among the Dagomba of Northern Ghana. Social Science and Medicine 50, 703-713.

Birungi, H. (1998) Injections and self-help: risk and trust in Uganda health care. Social Science and Medicine 47, 1455-1462.

Bledsoe, C; Banja, F. \& Hill A.G. (1998) Reproductive mishaps and Western contraception: An African challenge for fertility theory. Population and Development Review 24, 15-57.

Dein, S. (2007) Explanatory models and over systematization in medical anthropology. In R Littlewood (ed), On Knowing and Not Knowing in the Anthropology of Medicine. California: Left Coast Press.

Farmer, P. (1999) Infections and Inequalities: The Modern Plagues. Berkeley: University of California Press.

Feldman-Savelsberg, P., Ndoko, F.T. \& Schmidt-Ehry, B. (2000) Sterilizing vaccines or the politics of the womb: retrospective study of a rumour in Cameroon. Medical Anthropology Quarterly 14, 159-179. 
Garro, L. (1986) Decision-making models of treatment choice. In S. McHugh and T. Michael Valli (eds). Illness Behaviour: A Multi-disciplinary Model. New York: Plenum Press.

Hanson, K., Kikumbih, N., Amstrong-Schellenberg, J., Mponda, H., Nathan, R., Lake, S., Mills, A., Tanner, M. \& Lengeler, C. (2003) Cost-effectiveness of social marketing of insecticide-treated nets for malaria control in the United Republic of Tanzania. Bulletin of the World Health Organization 81, 269-276.

Hoppe, K.A. (1997) "Lord of the fly: colonial visions and revisions of African sleepingsickness environments on Ugandan Lake Victoria, 1906-61. Africa 67, 86-105.

Kjekshus, H. (1977) Ecology Control and Economic Development in East Africa History: The Case of Tanganyika 1850-1950. London: Heinemann Educational Books.

Leach, M. \& Fairhead, J. (2007) Vaccine Anxieties: Global Science, Child Health and Society. London: Earthscan.

Magesa, S.M., Lengeler, C., deSavigny, D., Miller, J.E., Njau, R.J.A., Kramer, K., Kitua, A. \& Mwita, A. (2005) Creating an "enabling environment" for taking insecticide treated nets to national scale: the Tanzanian experience. Malaria Journal 4, 34.

Maharaj, P. (2001) Male attitude to family planning in the era of HIV/AIDS: evidence from KwaZulu-Natal, South Africa. Journal of Southern African Studies 27, 245-257.

McCombie, S. (1996) Treatment seeking for malaria: A review of recent research. Social Science amd Medicine 43, 933-945.

Minja, H. \& Obrist, B. (2005) Integrating Local and Biomedical Knowledge and Communication: Experiences from KINET Project in Southern Tanzania. Human Organization 64,157-165.

Nichter, M. \& Nichter, M. (1996) Education by appropriate analogy. In M Nichter \& M Nichter, (eds). Anthropology and International Health: Asian Case Studies. Amsterdam: Gordon \& Breach.

Pell, C., Straus, L., Andrew, E.V.W., Meñaca, A., Pool, R. (2011) Social and cultural factors affecting uptake of interventions for malaria in pregnancy in Africa: A systematic review of the qualitative research. PLoS ONE 6, e22452. doi:10.1371/journal.pone.0022452

Pels, P. (1999) A Politics of Presence: Contacts between Missionaries and Waluguru in Late Colonial Tanganyika. Amsterdam: Harwood Academic Publishers.

Schellenberg, J.A., Minja, H., Mponda, H., Kikumbih, N., Mushi, A., Nathan, R., Abdulla, S., Mukasa, O., Marchant, T.J., Tanner, M. \& Lengeler, C. (2002) Re-treatment of mosquito nets with insecticide. Transactions of the Royal Society of Tropical Medicine and Hygiene 96, 368-369.

Vaughan, M. (1991) Curing Their Ills: Colonial Power and African Illness. Cambridge: Polity Press.

Wayland, C. (2004) The failure of pharmaceuticals and the power of plants: medicinal discourse as a critique of modernity in the Amazon. Social Science and Medicine 58, 2409-2419.

Whyte, S.R., Van der Geest, S. \& Hardon, A. (2002) Social Lives of Medicines. Cambridge: Cambridge University Press.

Winch, P.J., Makemba, A.M., Kamazima, S.R., Lwihula, G.K., Lubega, P., Minjas, J.N. \& Shiff, C.J. (1994) Seasonal variation in the perceived risk of malaria: implications for the promotion of insecticide-impregnated bed nets. Social Science and Medicine 39, 6375 . 
Winch, P.J., Makemba, A.M., Makame, V.R., Mfaume, M.S., Lynch, M.C., Premji, Z., Minjas, J.N. \& Shiff, C.J. (1997) Social and cultural factors affecting rates of regular retreatment of mosquito nets with insecticide in Bagamoyo District, Tanzania. Tropical Medicine E International Health 2, 760-770.

Wolf, D. (1985) The future of family planning in Sub-Saharan Africa. International Health Planning Perspectives 11, 1-8. 